\title{
Lean Blow-Out Prediction in Gas Turbine Combustors Using Symbolic Time Series Analysis
}

\author{
Achintya Mukhopadhyay* \\ Indian Institute of Technology Madras, Chennai 600 036, India \\ Rajendra R. Chaudhari,, \pm Tanoy Paul, \\ Jadavpur University, Kolkata 700 032, India \\ and \\ Asok RayI \\ Pennsylvania State University, University Park, Pennsylvania 16802 \\ DOI: $10.2514 / 1 . B 34711$
}

\begin{abstract}
This paper develops a novel strategy for prediction of lean blowout in gas turbine combustors based on symbolic analysis of time series data from optical sensors, where the range of instantaneous data is partitioned into a finite number of cells and a symbol is assigned to each cell. Depending on the cell to which a data point belongs, a symbolic value is assigned to the data point. Thus, the set of time series data is converted to a symbol string. The (estimated) state probability vector is computed based on the number of occurrence of each symbol over a given time span. For the purpose of detecting lean blowout in gas turbine combustors, the state probability vector obtained at a condition sufficiently away from lean blowout (reference state) is considered as the reference vector. The deviation of the current state vector from the reference state vector is used as an anomaly measure for early detection of lean blowout. The results showed that the rate of change of the anomaly measure with equivalence ratio changed significantly as the system approached lean blowout. This change in slope of the curve was observed approximately at a similar proximity to lean blowout for different operating conditions and, hence, could be used as an early lean blowout precursor. The actual location of the change of slope depended primarily on the choice of reference state. This technique performed satisfactorily over a wide range of premixing.
\end{abstract}

\section{Introduction}

$\mathbf{S}_{\mathrm{e}}^{\mathrm{T}}$ TRINGENT emission norms require gas turbines to operate with extremely low $\mathrm{NO}_{\mathrm{x}}$ production. Ultralean combustion is one commonly used approach for minimizing $\mathrm{NO}_{\mathrm{x}}$ production. However, ultralean combustion is extremely susceptible to thermoacoustic instabilities and lean blowout (LBO). LBO is extremely detrimental for both power station gas turbines and aeroengines. In land-based turbines, LBO leads to prolonged shutdown and relighting involving productivity loss. For aeroengines during throttling operation, the fuel flow is suddenly reduced. But, due to the inertia of the compressor, reduction in airflow takes place at a much slower rate. The consequent sudden decrease in equivalence ratio can lead to LBO on aircraft engines, which can have fatal consequences. A priori determination of the LBO margin may not be possible because it is dynamically altered in the presence of thermoacoustic instabilities. This calls for development of strategies for early detection of imminent blowout and adoption of appropriate measures to mitigate it. Although land-based gas turbines mostly operate in a lean premixed mode, in aircraft engines, the fuel is admitted close to the burner, leading to partially premixed combustion. The current paper

Received 21 June 2012; revision received 8 April 2013; accepted for publication 8 April 2013; published online 14 June 2013. Copyright (C) 2013 by Achintya Mukhopadhyay, Rajandra R. Chaudhari, Tanoy Paul, Swarnendu Sen and Asok Ray. Published by the American Institute of Aeronautics and Astronautics, Inc., with permission. Copies of this paper may be made for personal or internal use, on condition that the copier pay the $\$ 10.00$ per-copy fee to the Copyright Clearance Center, Inc., 222 Rosewood Drive, Danvers, MA 01923; include the code 1533-3876/13 and \$10.00 in correspondence with the CCC.

*Professor, Department of Mechanical Engineering, National Centre for Combustion Research and Development; also Mechanical Engineering Department, Jadavpur University, Kolkata, India; achintya.mukho@gmail .com (Corresponding Author).

${ }^{\dagger}$ Research Scholar, Department of Mechanical Engineering.

*Undergraduate Student, Department of Mechanical Engineering.

§Professor, Department of Mechanical Engineering.

Distinguished Professor, Department of Mechanical Engineering. examines strategies for mitigation of LBO for both premixed and partially premixed combustors.

A number of studies in the last few years attempted to identify LBO precursors with the objective of mitigating it. De Zilwa et al. [1] investigated the occurrence of blowout in dump combustors with and without swirl. Chaudhuri and Cetegen [2,3] investigated the blowoff characteristics of a bluff-body stabilized burner flame, representative of afterburners of gas turbine combustors in the presence of spatial gradients in mixture composition and velocity oscillations. They used photomultiplier tubes with $\mathrm{CH}^{*}$ chemiluminescence filters to capture the optical signal and characterized the signal in the vicinity of blowout. Chaudhuri et al. [4] and Stöhr et al. [5] used combined particle image velocimetry (PIV)-planar laser induced fluorescence (PLIF)-based diagnostics to explain the near LBO dynamics of premixed and partially premixed combustors. However, these works did not focus on developing strategies for mitigating LBO.

The LBO limit is dependent on a number of parameters related to the combustor configuration and operating conditions, and real-time monitoring for each of them requires a complicated and expensive diagnostic system. Quantifiable dynamic characteristics of flame preceding blowout has, therefore, been exploited in the past as LBO precursors. References [6-9] used time series data from acoustic and optical sensors for early detection and control of LBO in gas- and liquid-fuelled combustors. Use of both acoustic and optical sensors in the same combustor [9] demonstrated the superiority of optical sensors due to faster response time. Nair and Lieuwen [8] used spectral, statistical, wavelet-based, and thresholding-based techniques to detect blowout precursors. For statistical analysis, they used moving average kurtosis. For thresholding approach, they identified certain events like the peak pressure in a cycle falling below a certain threshold value. The number of such events was found to increase monotonically as the system approached extinction. For wavelet-based analysis, they used Mexican Hat wavelet and a customized wavelet that matches with the time series data of $\mathrm{OH}^{*}$ chemiluminescence. Yi and Gutmark [10] recommended two indices, namely, the normalized chemiluminescence root mean square and the normalized cumulative duration of LBO precursor events for LBO prediction in real time. However,

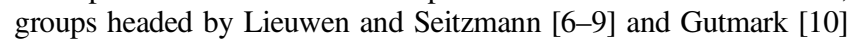


both demonstrated their techniques for LBO prediction in premixed combustors. The current work develops a novel technique based on symbolic time series analysis for LBO prediction in both premixed and partially premixed operating conditions.

Symbolic time series analysis (STSA) provides an elegant way of analyzing the dynamics of nonlinear systems [11]. Tang et al. [12] and Daw et al. [13] described the technique of generating symbolic time series by discretization of experimental or simulated time series. Rajagopalan and Ray [14], Subbu and Ray [15], Sarkar et al. [16], and Jin et al. [17] developed different procedures for generation of symbolic time series. They also developed a procedure for early detection of anomalous behavior in dynamical systems based on symbolic dynamic filtering (SDF) and applied it for detecting anomalous behavior in diverse physical systems [18-21]. Gupta et al. [22], Datta et al. [23], and Chakraborty et al. [24] applied SDF for predicting extinction in mathematical models of pulse combustors. Daw et al. [25] and Green et al. [26] used symbolic time series to characterize the dynamics of spark ignition engines. However, this procedure has not been in much use for early detection of anomalies using experimental data in combustion systems where measurement uncertainties and sensor noise invariably complicate the analysis. The objective of the present work is to explore the suitability of using symbolic time series analysis for early prediction of LBO in model gas turbine combustors. The work of Flynn et al. [27] on condition monitoring in flames has a similar objective although there are significant differences in details.

\section{Experimental Details}

Based on the earlier works of Williams et al. [28], Meier et al. [29], Nair et al. [6], Chaudhuri and Cetegen [2], and Yi and Gutmark [10], a swirl-stabilized dump combustor was designed as a laboratory-scale model of a generic gas turbine combustor, schematically shown in Fig. 1. The combustion air is supplied at ambient temperature from the compressor to the bottom port provided on the premixing tube, and metered upstream of the combustor using a calibrated mass flow controller (MFC) (Aalborg range 0-500 litres per minute (LPM)). The fuel (liquefied petroleum gas, LPG) is supplied from a pressurized cylinder fitted with a needle valve to control the flow rate and metered upstream of the combustor by a calibrated Aalborg mass flow controller (range 0-10 LPM.). To investigate the effects of premixing on flame dynamics, six side ports are provided $50 \mathrm{~mm}$ apart along the length of the premixing tube. This allows the fuel to be injected at different axial positions of the premixing tube, thereby providing different premixing lengths. The ports are numbered 1 to 6 from the bottom of the premixing tube. Thus, the fuel injected through port 1 allows greatest premixing whereas port 6 allows the least premixing. The fuel is injected to one of the side ports of the premixing tube. The fuel-air mixture entered the combustor through an inlet swirler in the annulus around a center body, located just prior to the dump plane in the premixing section. The inner diameter of the premixing section is $2.3 \mathrm{~cm}$, and the diameter of the center body is $0.8 \mathrm{~cm}$. The inlet swirler has six vanes positioned at $60 \mathrm{deg}$ to the flow axis. A quartz tube is provided in the combustion zone having an internal diameter of $6 \mathrm{~cm}$ and length $20 \mathrm{~cm}$ to facilitate optical diagnostics.

The heat release rate is measured by the chemiluminescence emitted from the $\mathrm{CH}^{*}$ radicals $(\lambda \sim 431 \mathrm{~nm})$ of the flame. The time series data are obtained with a photomultiplier tube (PMT) fitted with an optical band pass filter $\left(\lambda_{\text {pass }}=430 \mathrm{~nm}\right.$, full wave half width (FWHM) $10 \mathrm{~nm}$ ). The PMT output signal (voltage) is acquired using a 16-bit analog input channel on a National Instruments PXI-6250 data acquisition card mounted in a National Instruments PXI-1050 chassis having a built-in 08 channel SCXI-1125 signal conditioner module. A time series of $2^{15}$ data points is acquired at a sampling frequency of $2 \mathrm{kHz}$ in each experiment using National Instruments Lab VIEW® 7.1. Video images of the flame are recorded in order to visualize LBO phenomenology and correlate the same with the optical signal. Still color images of the flame are also acquired simultaneously using the digital single lens reflex camera at suitable exposure to avoid pixel saturation. Further details of the experimental setup and instrumentation are available in Chaudhari [30].

Experiments were carried out using LPG as fuel. A major reason for choice of LPG as fuel was that LPG consists of mainly propane and butane, which are the simplest hydrocarbons whose combustion exhibits chemical behavior, flame speed, and extinction limits closer to the heavier and more complex hydrocarbon fuels [31]. The experiment involved first using stoichiometric air-fuel mixture $(\Phi=1)$. Then, at a given airflow rate, the fuel supply is gradually decreased to generate progressively lean reacting mixtures. Because the air constitutes the bulk of the incoming reactant mixture, a constant airflow rate ensures that the flow time remains practically constant.

\section{Symbolic Time Series Analysis}

The procedure for anomaly detection using STSA has been described in detail by Ray [11], Rajagopalan and Ray [14], and Jin et al. [17]. Here it will be only briefly reviewed.

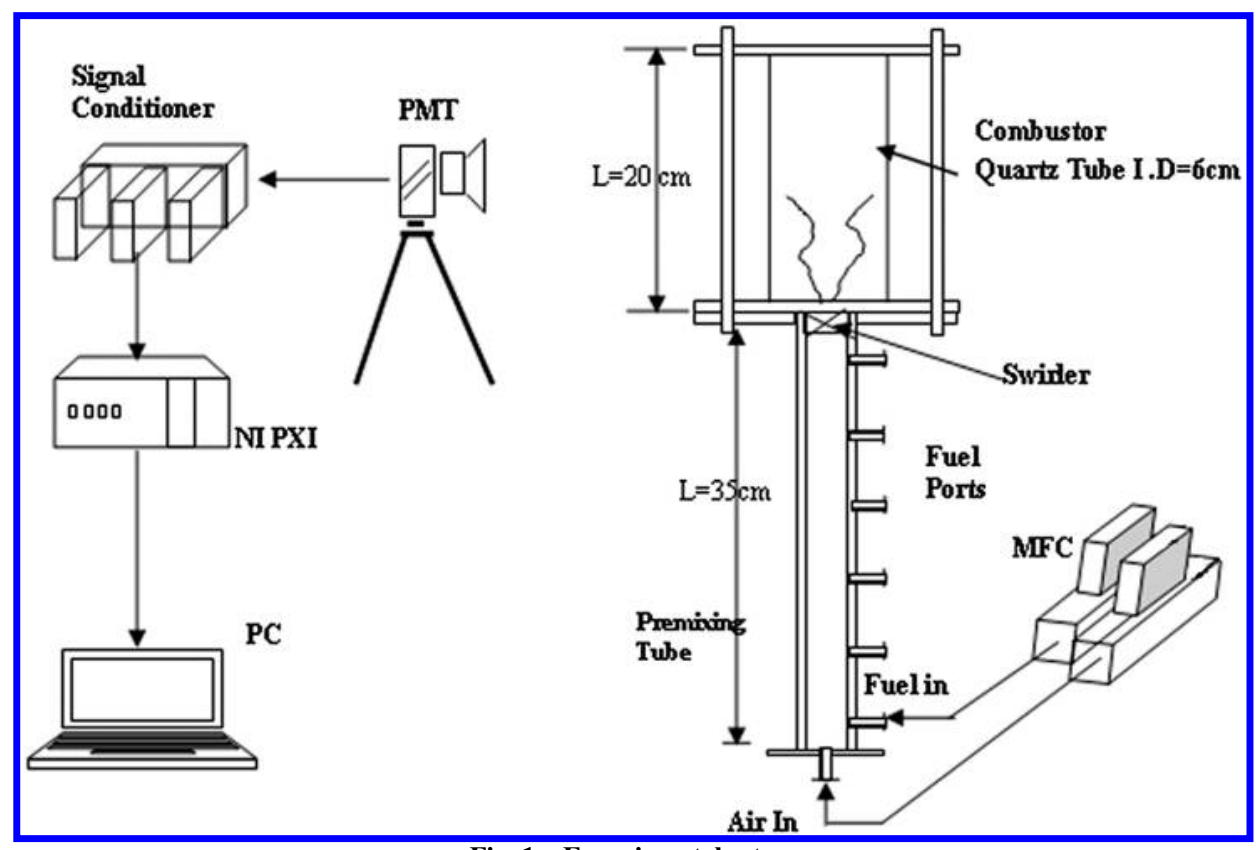




\section{A. Generation of Symbolic Time Series}

STSA encodes the behavior of (possibly nonlinear) dynamical systems from the preprocessed time series by symbolization and state machine construction. This is followed by computation of the state probability vectors that are representatives of the evolving statistical characteristics of the dynamical system.

Figure 2 outlines the concept of STSA to encode the dynamical behavior of systems from observed time series data, state machine construction, and computation of the state probability vectors that are representatives of the evolving characteristics of the dynamical system. The ensemble of the time series data is partitioned into a mutually exclusive and exhaustive set of finitely many segments. Let each segment in the plot at the upper left-hand corner of Fig. 2 be labeled by a unique symbol, and let $\Sigma$ denote the alphabet of all these symbols. The segment, visited by the time response plot at a (fast scale) instant, takes a symbol value from the alphabet $\Sigma$. For example, in Fig. 2, $\Sigma=\{\alpha, \beta, \gamma, \delta\}$ and a sensor time series $x_{0} x_{1} x_{2} \cdots$, generates a string of symbols in the symbol space as $s_{0} s_{1} s_{2} \cdots$, where each $s_{i}, i=0,1,2, \cdots$ takes a symbol value from the alphabet $\Sigma$. This mapping is called symbolic dynamics because it attributes a legal (i.e., physically admissible) symbol string to the dynamical system starting from an initial state. For example, see the symbol string at the top right-hand corner of Fig. 2.

It is evident from the preceding discussion that a crucial step in STSA is the generation of a symbol string by appropriately partitioning symbolic time series analysis. For generation of symbolic time series in the present application, the entire range of data for a particular measurement (e.g., heat release rate fluctuation) is partitioned into a finite number of nonoverlapping contiguous cells. The unique symbol is assigned to every instantaneous value of the measured variable that falls in that partitioned region. Thus, the discrete time series of the state variable is converted to a symbol string (cf. Fig. 2). The time series data can be partitioned for symbol string generation using uniform partitioning or maximum entropy partitioning. In the present work, partitions are generated in such a way that, at an equivalence ratio away from LBO (designated the reference state), the number of occurrences of each symbol in the symbol string (i.e., number of data points in each partitioned region) is equal. This maximizes the information entropy

$$
H(k)=-\sum_{i=1}^{i=k} p_{i} \log p_{i}
$$

where $p_{i}$ denotes the probability of occurrence of a symbol $\sigma_{i}$ in the string. Symbolic time series are generated in this work in two different ways. In one method, known as simple partitioning (SP), the time series data are directly used for the partitioning. The other technique used was analytic signal space partitioning (ASSP) [15]. This technique uses complex valued analytic function $A[\bar{x}](t)$ corresponding to the time series data, defined as

$$
A[x](t)=x(t)+i \tilde{x}(t)=R(t) \exp (i \phi(t))
$$

where $\tilde{x}(t)$ is the Hilbert transform of the time series data and $R(t)$ and $\phi(t)$ are the magnitude and the phase of the complex valued analytic function. The magnitude and the phase of the analytic function are partitioned separately using maximum entropy partitioning. Thus, each data point is represented by a pair of symbols, one belonging to the symbol string $\Sigma_{R}$ (based on the magnitude) and the other belonging to the symbol string $\Sigma_{\phi}$ (based on the phase). The analytic signal is partitioned into a symbol string by associating each pair of symbols into a symbol from a new alphabet $\Sigma$ as

$$
\Sigma=\left\{\left(\sigma_{i}, \sigma_{j}\right): \sigma_{i} \in \Sigma_{R} ; \sigma_{j} \in \Sigma_{\phi}\right\} \quad \text { and } \quad|\Sigma|=\left|\Sigma_{R}\right|\left|\Sigma_{\phi}\right|
$$

Further details of Hilbert-transform-based ASSP are reported by Subbu and Ray [15].

\section{B. Construction of Probabilistic Finite State Automata}

The core assumption in STSA for construction of probabilistic finite state automata (PFSA) from symbol strings is that the symbolic process under both nominal and fault/damage conditions can be approximated as a Markov chain of order $D$, called the $D$-Markov machine, where $D$ is a positive integer. Although the details of the $D$-Markov machine are given in [11], the pertinent information on the construction of a $D$-Markov machine is presented next.

A $D$-Markov chain is a statistically (quasi-)stationary stochastic process $S=\cdots s_{-1} s_{0} s_{1} \cdots s_{n} \cdots$, where the probability of occurrence of a new symbol depends only on the last $D$ symbols:

$$
p\left(s_{n} \mid s_{n-1} \cdots s_{n-D} \cdots s_{1}\right)=p\left(s_{n} \mid s_{n-1} \cdots s_{n-D}\right)
$$

The construction of a $D$-Markov machine is based on 1) state splitting that generates symbol blocks of different lengths according to their relative importance, and 2) state merging that assimilates histories from symbol blocks leading to the same symbolic behavior [32].
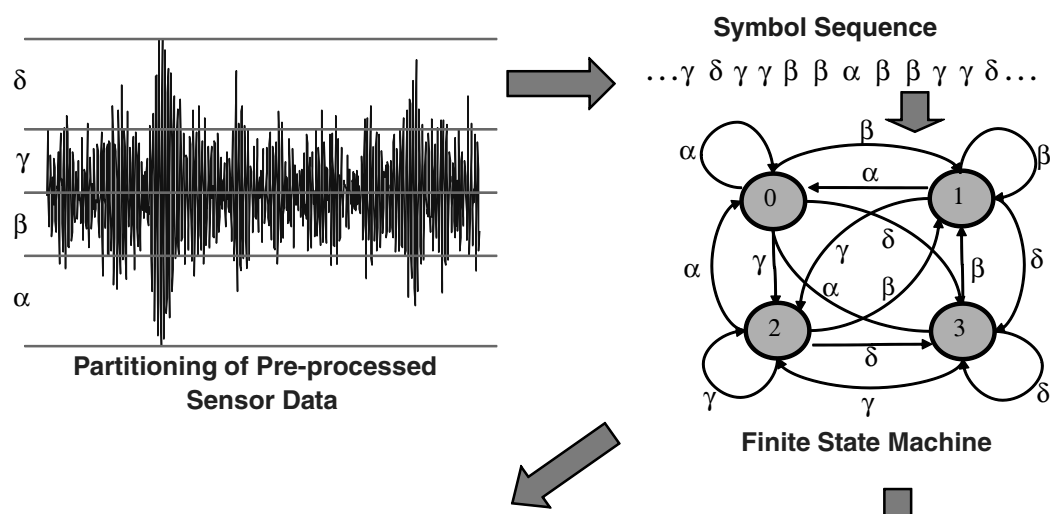

Finite State Machine
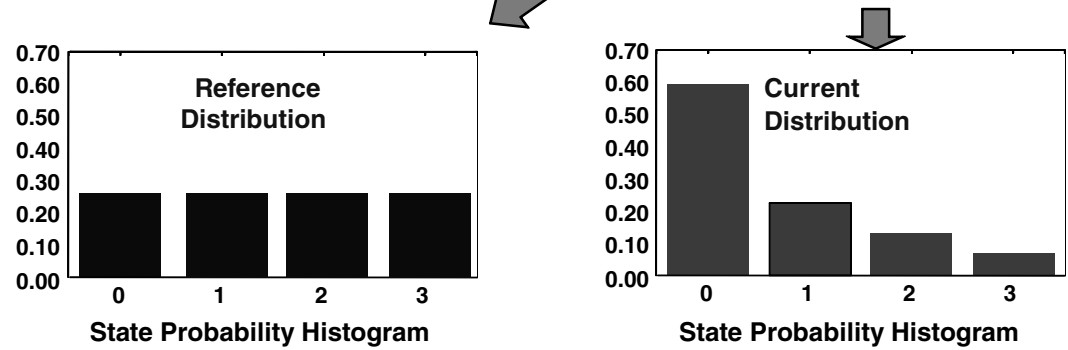

Fig. 2 Concept of symbolic time series analysis. 

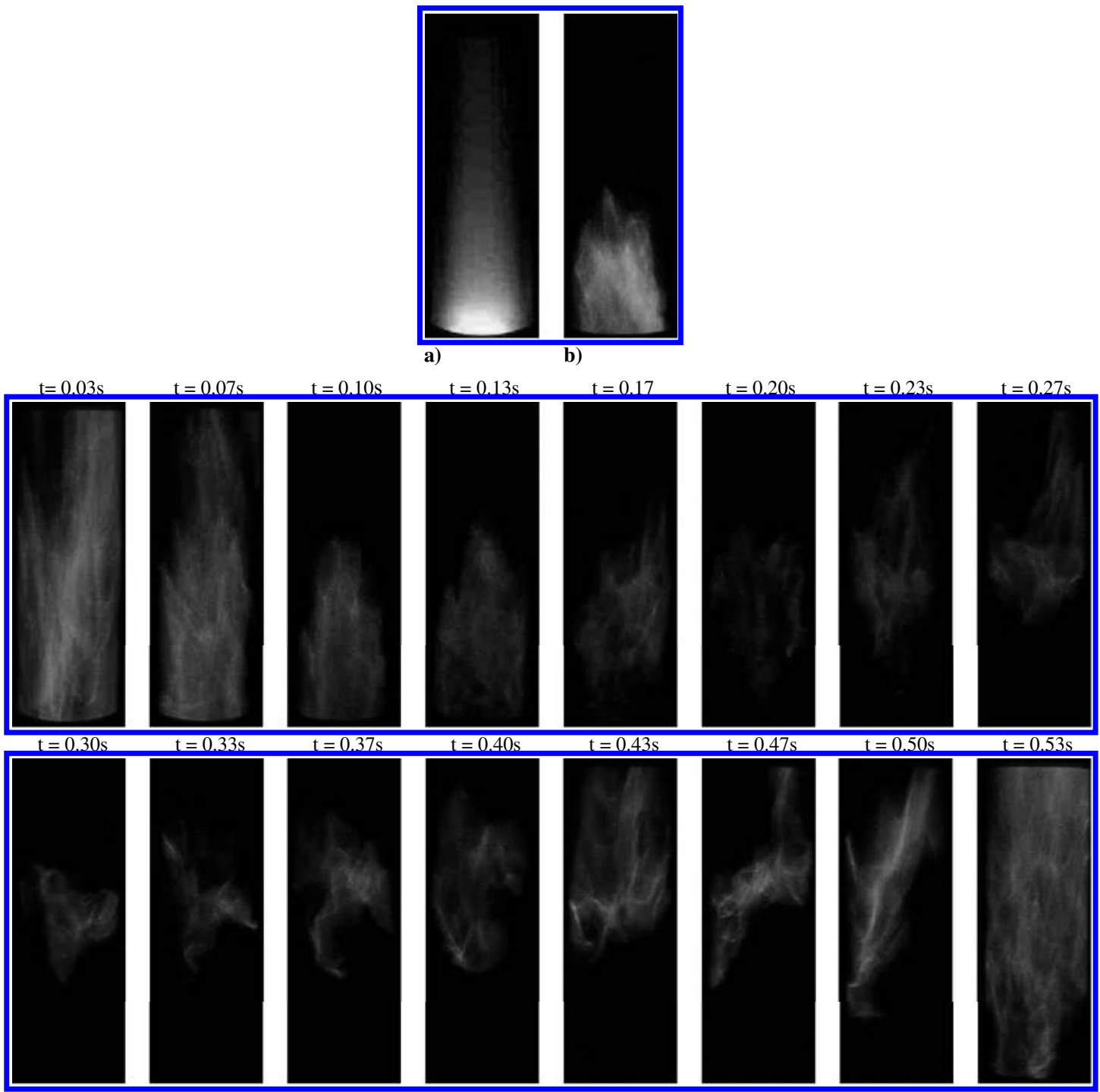

Fig. 3 Flame images for fuel injected through port $1\left(L_{\mathrm{fuel}}=35 \mathrm{~cm}\right)$ for a) $\left.\phi=1.0, \mathrm{~b}\right) \phi=0.81$, and c) $\phi=0.75$.
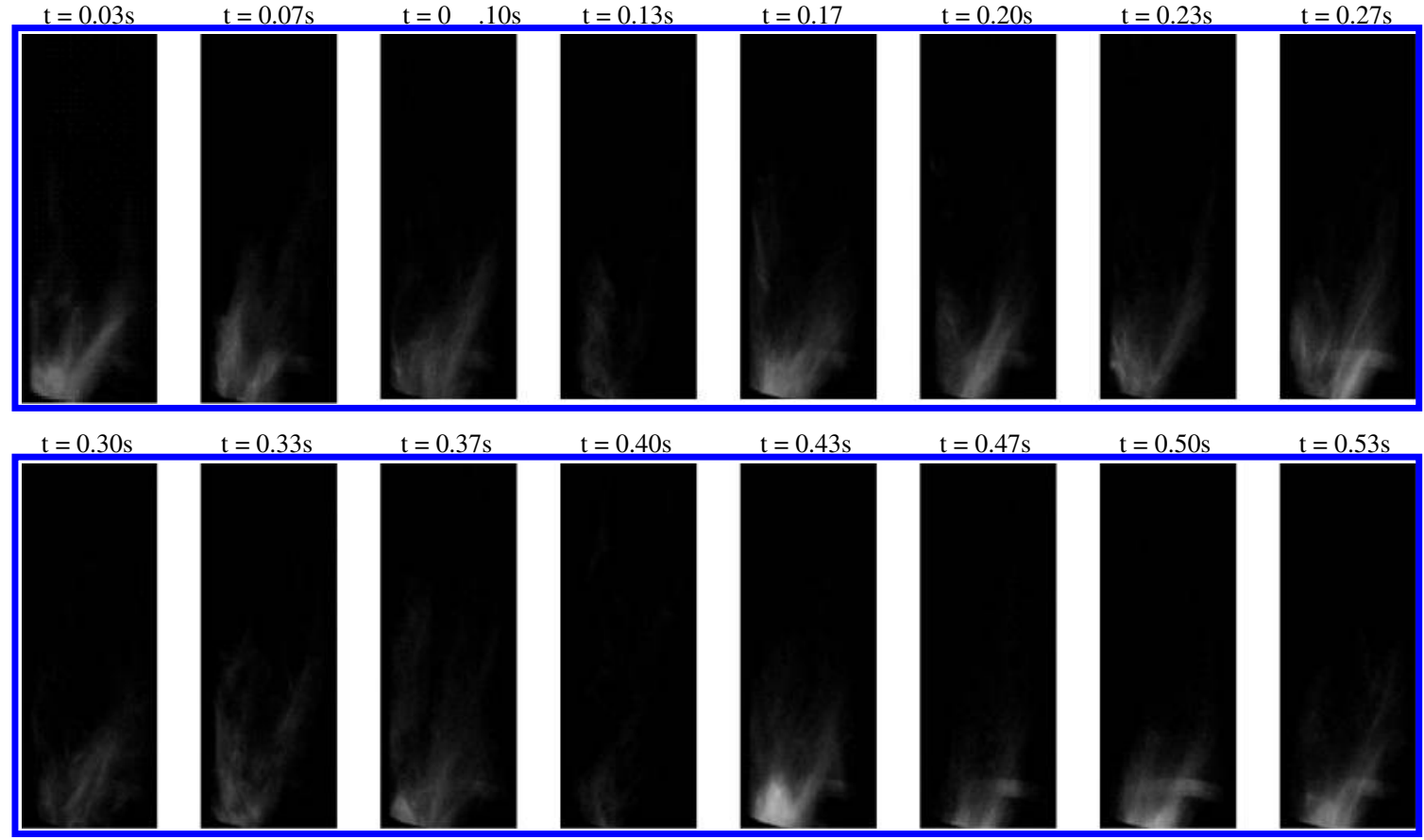

Fig. 4 Flame images for fuel injected through port $5\left(L_{\mathrm{fuel}}=15 \mathrm{~cm}\right)$ for $\phi / \phi_{\mathrm{LBO}}=1.0$. 
Words of length $D$ on a symbol string are treated as the states of the $D$-Markov machine before any state merging is executed. Thus, on a (finite) alphabet $\Sigma$ with cardinality $|\Sigma|$, the total number of possible states is less than or equal to $|\Sigma|^{D}$, and operations of state merging may significantly reduce the number of states. As the value of $D$ is increased, more memory is imbedded in the Markov states of the PFSA. However, the benefits of having additional memory associated with a larger value of $D$ could be offset by the increased computational load. Therefore, one must make a tradeoff between the two competing requirements of 1) capturing information from time series, and 2) reduction of computational complexity in terms of memory requirements and execution time in the construction of $D$-Markov machine algorithms.

In the present work, the word size $D$ is taken to be 1 and, hence, the number of possible states is equal to the number of symbols; therefore, the results reported in this paper are limited to $D=1$. However, in general, larger values of the integer parameter $D$ (i.e.,
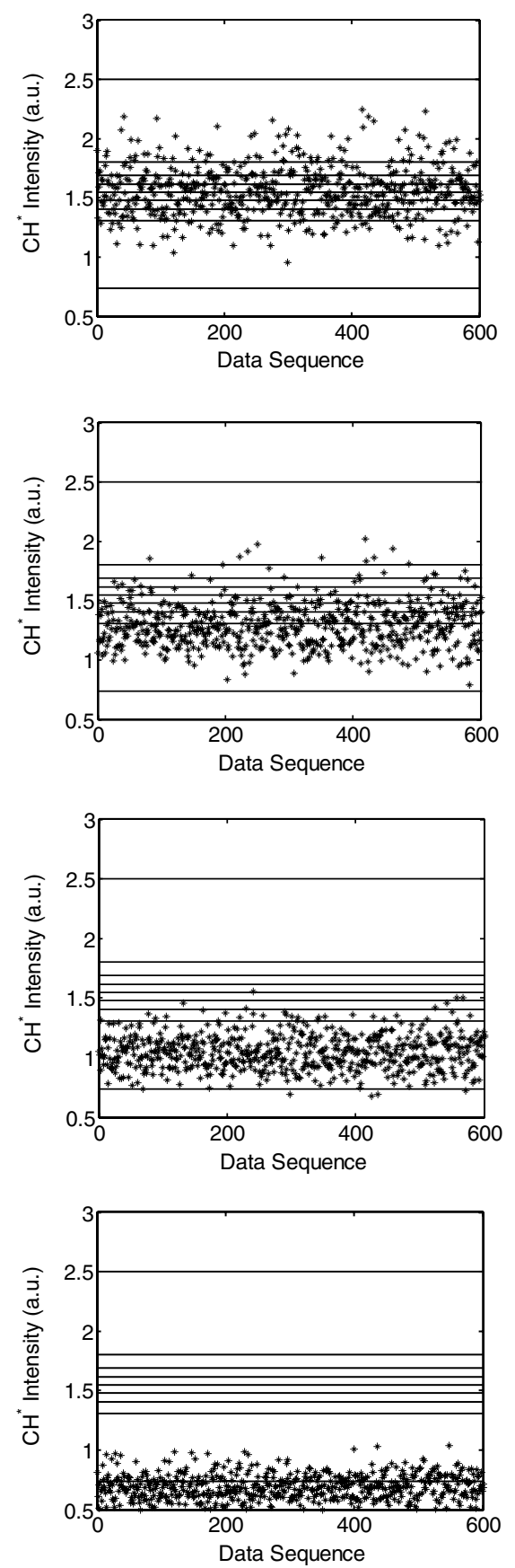
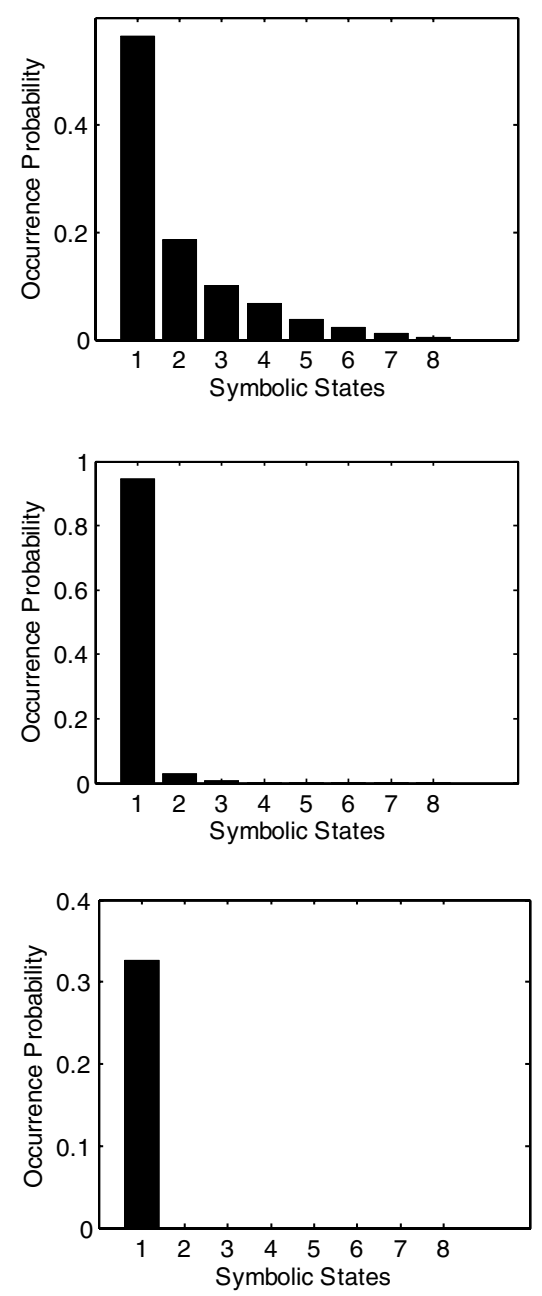

$D>1$ ) might be necessary to capture the long time-scale information about combustion fluctuations near LBO. It is noted that the operations of state splitting and state merging [32] might be necessary for PFSA construction with $D>1$, whereas these operations are not required for $D=1$.

\section{Construction of Anomaly Measure}

Following Fig. 2, sensor time series at a reference state (e.g., equivalence ratio) $\phi_{k}$ may now be used to compute the state probabilities $p_{j}^{k}$; that is, the probability of being in the state $j$ at time reference state $\phi_{k}$. The state probability vector is obtained as $\boldsymbol{p}^{k}=\left[p_{1}^{k}, \ldots, p_{n}^{k}\right]$, where $n$ is the number of PFSA states. In the present case, the number of PFSA states is equal to the alphabet size as $D=1$. At another state $\phi_{\ell}$, distinct from the reference state, the state probability vector $\boldsymbol{p}^{k}$ changes to $\boldsymbol{p}^{\ell}$. The state probability vector can be used for computation of a scalar anomaly measure $d\left(\boldsymbol{p}^{k}, \boldsymbol{p}^{0}\right)$ as the distance of the current probability vector $p^{k}$ from the

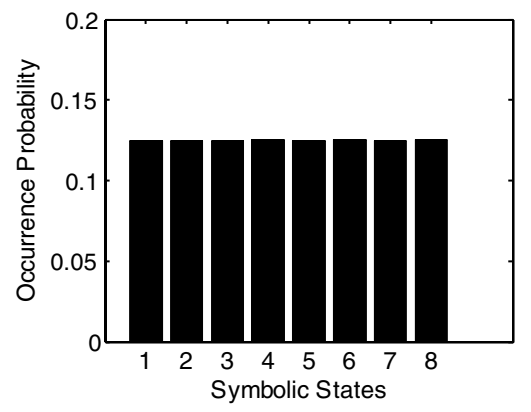

Fig. 5 Time series data and symbolic state histograms at $\phi=0.9,0.85,0.8$, and 0.75 for port 1 with airflow $Q=70 \mathrm{lpm}$. 
probability vector $\boldsymbol{p}^{0}$ at the nominal condition denoted by the superscript 0 . Two histograms at the bottom of Fig. 2 illustrate $\boldsymbol{p}^{0}$ and $\boldsymbol{p}^{k}$. In this paper, the anomaly measure is defined as

$$
M=\operatorname{Cos}^{-1} \frac{<\boldsymbol{p}^{k}, \boldsymbol{p}^{0}>}{\left\|\boldsymbol{p}^{k}\right\|\left\|\boldsymbol{p}^{0}\right\|}
$$

where $<\boldsymbol{p}^{k}, \boldsymbol{p}^{0}>$ denote the inner product of the state probability vectors $\boldsymbol{p}^{k}$ and $\boldsymbol{p}^{0}$, and $\|\boldsymbol{p}\|$ denotes the Euclidean norm of the vector $\boldsymbol{p}$. This anomaly measure is used as an LBO detection metric.

Rao et al. [33] have reported the superior performance of STSA in comparison to other classes of pattern recognition tools, such as Bayesian filters and artificial neural networks, from the perspectives of 1) anomaly detection capability, 2) decision making for failure mitigation, and 3) computational efficiency. From these perspectives, the major advantages of STSA for anomaly detection are listed.

1) The method is robust to measurement noise and spurious signals.

2) The technique is adaptable to low resolution sensing due to coarse graining in space partitions.

3 ) The method is capable of early detection of anomalies because of sensitivity to signal distortion and real time execution on commercially available inexpensive platforms.

4) The technique is computationally efficient in terms of both execution time and memory because no large-order recursive equations need to be solved.

\section{Results and Discussions}

Experiments were carried out using LPG at six airflow rates. Three sets of results were generated at relatively low airflow rates of 70, 80, and $85 \mathrm{lpm}$. Another set of results was generated for high airflow rates of 150, 175, and $200 \mathrm{lpm}$. The corresponding Reynolds number based on cold flow conditions varies from 6545 (corresponding to an airflow rate of $70 \mathrm{lpm}$ ) to 18,700 (corresponding to an airflow rate of $200 \mathrm{lpm})$.

\section{A. Experimental Observations}

Figure $\underline{3}$ shows the flame images for fuel injected through port 1 (distance of fuel injection port from the dump plane, $L_{\text {fuel }}=35 \mathrm{~cm}$ ), which gives the maximum premixing of air and fuel for equivalence ratios far from LBO $(\Phi=1.0$ and 0.81$)$ and very close to $\mathrm{LBO}$ $(\Phi=0.75)$, where $\Phi$ is the air-fuel mixture quality. In the case of stoichiometric air-fuel mixture (i.e., $\Phi=1.0$ ), the combustion is seen to be relatively steady and occupies the whole combustor, and the shape of the flame is observed to be conical (Fig. 3a). As the equivalence ratio is reduced, for the $\Phi=0.81$ case, there is significant change in color of flame: the flame becomes bluish with a reddish tip. Although the flame zone changes from image to image, there is always a well-defined combustion region and the flame looks stable (Fig. 3b). Figure 3c plots a sequence of images captured at 30 frames per second for an equivalence ratio close to LBO; that is, $\Phi=0.75$.

For conditions close to blowout $\left(\Phi_{\mathrm{LBO}}=0.75\right)$, the flame shape changes from conical to columnar and the length increases due to reduced reaction rate and burning velocity of the flame near LBO. Near LBO limit, there are random instances where the flame exhibits oscillation in the combustor and flame liftoff from the dump plane. These oscillations may produce a near-flame-loss event in the combustor. The combustor initially has a spatially compact combustion zone. Then the flame detaches from the center body, showing weak reaction and moves farther downstream from the combustor inlet and stabilizes there. From downstream, the flame packets are convected back to inlet, which reignites the unburned fuel that entered the combustor in the previous period. The fuel is rapidly consumed, which exhibits intense combustion, and the flame is reestablished in the combustor (reignition event). These unique extinction and reignition events span a period of several milliseconds, and they occur randomly in time prior to LBO.
Figure 4 shows the images of the flame at $\Phi / \Phi_{\mathrm{LBO}}=1$ for the port 5 case, where the premixing length is minimum $L_{\text {fuel }}=15 \mathrm{~cm}$. The low premixing length for fuel-air mixing ensured that the flame obtained in the combustor is with lower degree of fuel-air premixing or in mostly partially premixed condition. From Figure 4 , it is revealed that, at all times, the flame is attached to the dump plane and does not show any liftoff pattern. Although the flame shows some flame loss events where the flame intensity is significantly reduced and there is subsequent reignition of flame, the flame does not oscillate in the combustor, and so the precursor events are not so intense. The unsteadiness is observed in the flame front in terms of flame flickering, which is clearly observed in images and the flame base is attached to the dump plane all the times, which is in contrast with the observation of port 1 flame characteristics. The flame is not symmetrically attached to the dump plane and exhibits asymmetric spread in the combustion chamber.

\section{B. Symbolic State Vectors}

Based on several trials, the number of symbolic states is fixed at eight for the simple partitioning. For ASSP, the magnitude space is partitioned into eight states and the phase space into five states. For both the cases, maximum entropy partitioning is followed for the reference state. For other states, the partitions are kept unchanged. Figure 5 shows the time series of $\mathrm{CH} *$ chemiluminescence recorded by the photomultiplier tube (PMT) fitted with a narrow bandpass filter, centered around $430 \mathrm{~nm}$ and the probability of occurrence of the symbolic states with simple partitioning for fuel injection through port 1 as the system approaches LBO. The horizontal lines superposed on the time series data indicate the partitions used for generation of symbols. From the time series data, it is observed that,

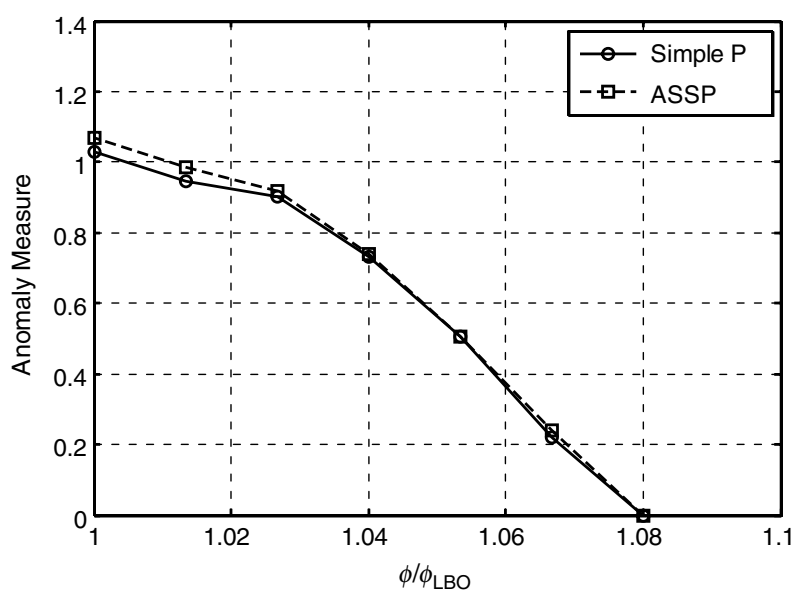

a)

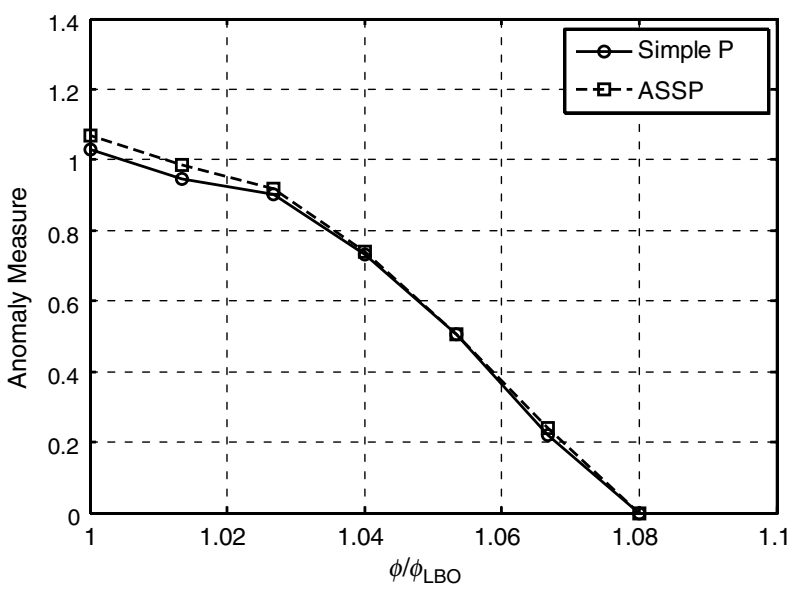

b)

Fig. 6 Variation of anomaly measure with $\phi / \phi_{\mathrm{LBO}}$, window size $=$ $13 \mathrm{~s}$ a) $1 \mathrm{~s}$ b). 
as the equivalence ratio is reduced, the intensity falls to lower values. Because the symbol partitions are kept unaltered, as explained in the preceding section, most of the data points now lie in the lower partitions. Thus, the histogram of the probability of the occurrence of the symbolic states gets skewed toward the lower ranges. Qualitatively similar results are obtained with simple partitioning and ASSP. The trend of the intensity decreasing with decrease in equivalence ratio is observed for other levels of premixing also, which leads to a similar change in the pattern of the probability histograms for all levels of premixing as LBO is approached. The difference in the patterns of the histograms at the reference and near LBO states is reflected in the anomaly measure, which is used for LBO detection. The present approach, thus, depends more on the overall behavior of the system, which responds similarly to decrease in equivalence ratio at all levels of premixing. Thus, the present approach is suitable for LBO detection at different levels of premixing, as the results in the next section show.

\section{LBO Prediction}

Figure 6 a shows the anomaly measures obtained for fuel injection through port $1\left(L_{\text {fuel }}=35 \mathrm{~cm}\right)$ using the two approaches: simple partitioning and ASSP. Earlier studies [15] revealed that ASSP gives more accurate prediction of approaching anomalies. On the other hand, ASSP requires a considerable amount of postprocessing of data compared to simple partitioning, which can practically work with the sensor data itself. This is especially important in the context of LBO, where the dynamics are fast, and real-time detection and control requires fast computation. The results, however, reveal that, in the present case, both the approaches produce results that are similar both qualitatively and quantitatively. The general trend shows that, at first, the anomaly measure increases sharply but, as LBO is approached, the measure becomes nearly constant. This can be explained with a reference to Fig. 5. As the equivalence ratio decreases, the PMT reading also decreases. Consequently, only the lowest partitions remain populated at lower equivalence ratios, leading to similar anomaly measures close to blowout. For real-time prediction of LBO, the size of data sampling window is also important because it directly affects the processing time. A comparison of Figs. $\underline{6 a}$ and $\underline{6 b}$ shows that the method works successfully even with a data sampling window of $1 \mathrm{~s}$.

Figure 7 shows the performance of this technique using simple partitioning in predicting LBO for different flow rates and fuel-air premixing. For all the flow rates and fuel inlet positions considered, the anomaly measure becomes practically constant as LBO is approached. Thus, this sudden decrease in the slope of the curve can be considered a precursor of LBO. Moreover, this change in slope occurs at $\Phi / \Phi_{\mathrm{LBO}}=1.2-1.4$. From the viewpoint of control implementation, another significant observation is that the nearly constant value of the anomaly measure is in the range of 1.1-1.2 for all the cases investigated. It may here be noted that, in all these experiments, the reference cases were taken to be close to stoichiometric.

Figure 8 shows the corresponding results with ASSP. It is observed that the qualitative behavior is identical with Fig. 7 although quantitative variation exists. Because simple partitioning involves less computation, it is recommended in the present work. The results of both Figs. 7 and $\underline{8}$ indicate that the technique is versatile enough for wide levels of premixing. This is in contrast with the methods of LBO detection in published literature, which is shown to work primarily for premixed flames only.

The LBO limit widely varies with the extent of premixing. Consequently, the proximity of the reference state from the LBO limit also changes considerably. Because it has been observed earlier that

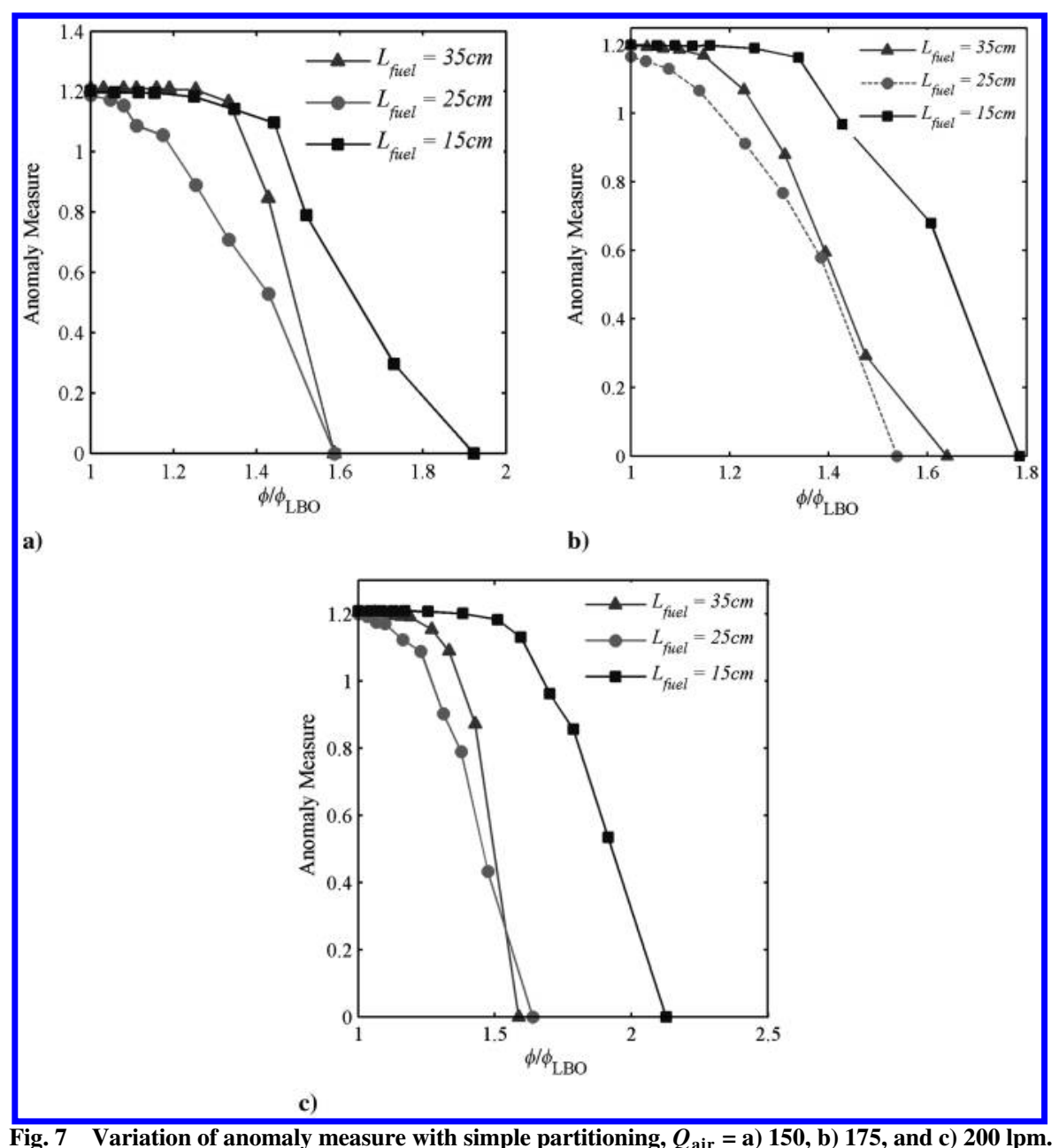



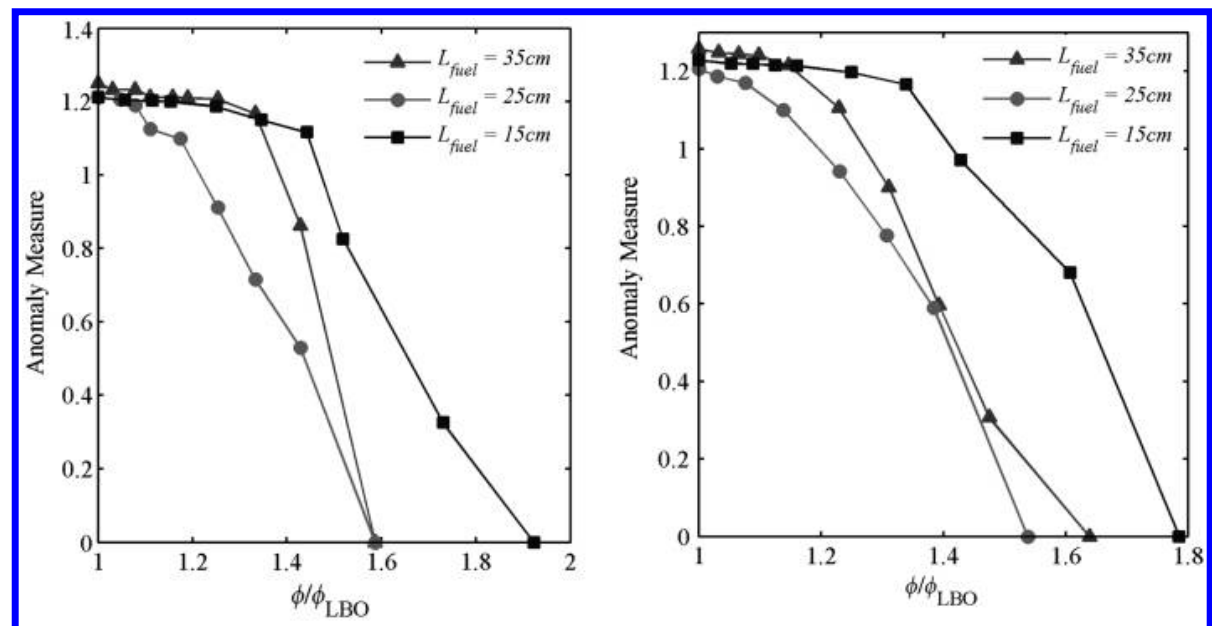

a)

b)

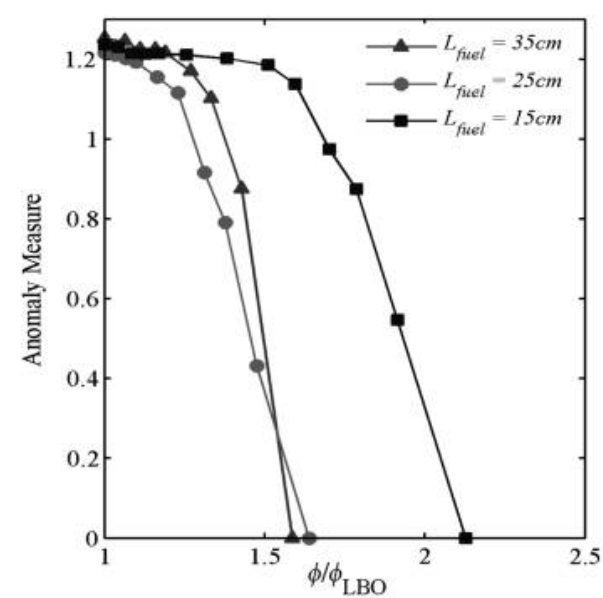

c)

Fig. 8 Variation of anomaly measure with ASSP, $Q_{\text {air }}=$ a) 150, b) 175, and c) $200 \mathrm{lpm}$.

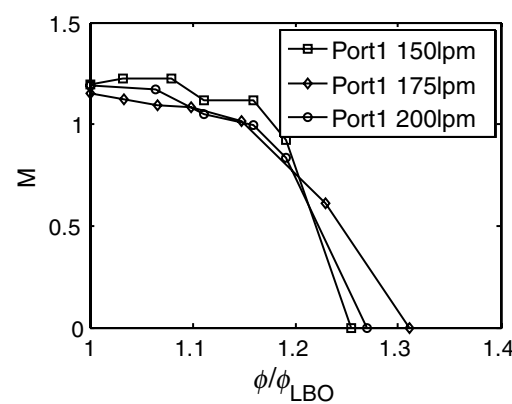

a)

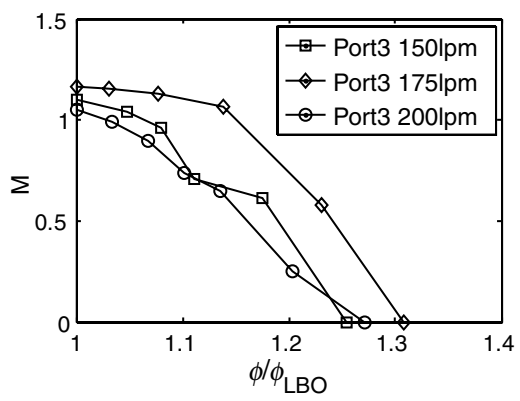

b)

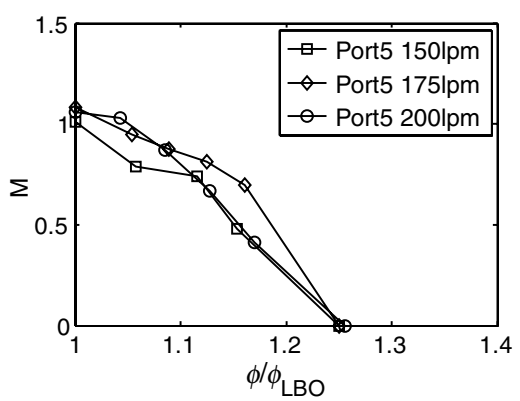

c)

Fig. 9 Variation of anomaly measure calculated by simple partitioning with $\Phi / \Phi_{\mathrm{LBO}}$ for different port position $\left(L_{\mathrm{fuel}}\right)$ and airflow $Q_{\text {air }}$ : a) port 1 , b) port 3 , and c) port 5 . 
the results are sensitive to the choice of reference state, the range of $\Phi / \Phi_{\mathrm{LBO}}$ where the anomaly curve changes slope and predicts LBO can be considerably reduced by choosing the reference state at a fixed proximity to the LBO threshold. Moreover, the high value of $\phi / \phi_{\mathrm{LBO}}$ where the slope changes in Figs. 7 and 8 would lead to operating the combustor at a much richer equivalence ratio than desired. This can be remedied by choosing the reference state at approximately $\phi / \phi_{\mathrm{LBO}}=1.25$. Figure 9 shows the anomaly measures with simple partitioning for such reference states. It is observed that the change of slope takes place at a much lower equivalence ratio at about $\phi / \phi_{\mathrm{LBO}}=1.1-1.15$. This allows operation at a much leaner limit. However, this approach requires at least an approximate prior knowledge of $\phi_{\mathrm{LBO}}$.

\section{Implications for Real-Time Control}

The results presented in the earlier subsections could lead to a strategy for real-time control schemes for mitigating LBO. The efficacy of real-time control would be enhanced by reducing the extent of online computation, which can be achieved by computing a significant part of the present algorithm a priori. For example, the generation of the partition and the symbolic state vector for the reference condition can be computed offline prior to commencement of the online operation. Thus, the only part of the algorithm that requires online computation is the generation of the symbolic state at the actual operating point and computation of the anomaly measure. The simple partitioning involves only segregating the data into proper bins and, hence, is recommended as a suitable technique for controlling LBO. Data sampling window can be optimally selected as a tradeoff between accuracy and computation time [14].

\section{Conclusions}

A novel strategy for detection of lean blowout (LBO) based on optical sensor data was developed and applied in a laboratory-scale swirl-stabilized dump combustor. The strategy involved generation of symbolic time series. In symbolic time series analysis, the time series data from the photomultiplier tube (PMT) is converted to a symbol string by partitioning the measurement range of the PMT sensor into a finite number of cells and assigning a symbol to each cell, depending on the cell in which its measured value falls. Symbol blocks of length $D$ are treated as the states of the probabilistic finite state machine (PFSA). In this paper, the symbol block parameter for PFSA states is selected to be $D=1$. However, in general, larger values of the integer parameter $D$ (i.e., $D>1$ ) might be necessary to capture the long-time-scale information on combustion fluctuations near LBO. In that case, the number of states would increase with the upper limit of $|\Sigma|^{D}$, where $|\Sigma|$ is the cardinality of the alphabet $\Sigma$ in the PFSA; consequently, tools of state splitting and state merging might be necessary to reduce the number of states in the PFSA construction. Thus, an important topic of future research is investigation of combustion processes with a relatively long memory, where the PFSA may have to be constructed from symbol strings with $D>1$ by making use of the tools of state splitting and state merging.

The array of the probabilities of occurrence of data in each partition cell constitutes the state vector for a given state. Keeping the partitions fixed, the state vectors are generated for different states and compared with those of a reference state, away from LBO. The difference between the vectors constitutes an anomaly measure. This measure is used as an LBO detection metric.

In the present work, the time series data of $\mathrm{CH}^{*}$ chemiluminescence is used to generate a symbolic time series using simple partitioning and analytic signal space partitioning, both based on maximum entropy approach such that all states are equiprobable at the reference state. The results show that both simple and analytic signal space partitioning give similar results. The anomaly measure first increases rapidly as the equivalence ratio is decreased but approaches a constant value as LBO is approached. This behavior is observed for a wide range of flow rates and air-fuel premixing. Thus, the change in slope can be used for LBO detection at various levels of premixing, making the method more versatile than those commonly reported in literature mainly for premixed flames. Moreover, in all the cases, the anomaly measure approaches a constant value within a narrow range for different levels of premixing. The combustor can be operated at a closer proximity to LBO by choosing the reference state at a fixed distance from LBO.

\section{Appendix: Algorithms for Anomaly Detection}

The algorithms for anomaly detection using simple partitioning and analytic signal space partitioning (ASSP) are given next.

The simple partitioning algorithm is described as follows: Generation of partitions

1) Generate time series data of length $N, x(i)$ for the nominal state.

2) Select number of states $N_{1}$ and assign one symbol to each partition (alphabet).

3) Arrange the time series data in ascending order and store the sorted series as $x 1(i)$.

4) Assign $N_{2}=\left\lfloor\frac{N}{N_{1}}\right\rfloor$ where \lfloor\rfloor denotes nearest lower integer.

5) Assign partition $(1)=x 1(1)$ and $\operatorname{partition}(i+1)=x 1\left(i * N_{2}\right)$ for $1 \leq i \leq\left(N_{1}-1\right)$ and partition $\left(N_{1}+1\right)=x 1(N)$

Construction of state probability vector for nominal state

1) Initialize state probability vector $p(i)=0$ for $1 \leq i \leq N_{1}$

2) for $j=1: N$

for $i=1: N_{1}$ if $x(j) \geq \operatorname{partition}(i)$ and $x(j)<\operatorname{partition}(i+1)$,

$$
p(i)=p(i)+1
$$

end end

end

3) $p 0(i)=p(i) / N$ for $1 \leq i \leq N_{1}$

Construction of state probability vector for other states

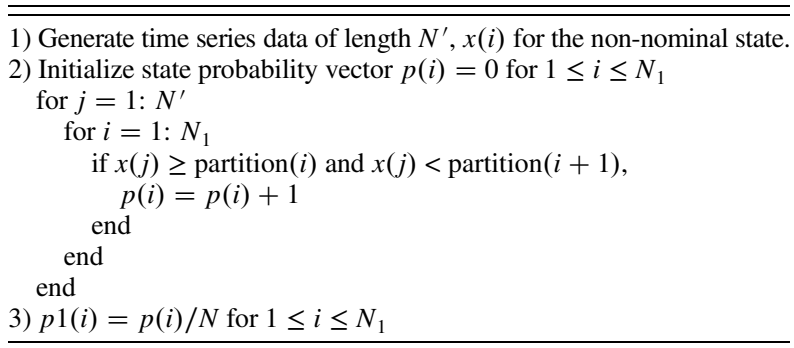

Use the same partitions for all the states.

Determination of anomaly measure

$\overline{\overline{1) \text { Compute anomaly measure, } M=\operatorname{Cos}^{-1} \frac{\boldsymbol{p}_{0} \cdot \boldsymbol{p}_{1}}{\left\|\boldsymbol{p}_{0}\right\|\left\|\boldsymbol{p}_{1}\right\|}}}$

The ASSP algorithm is described as follows:

Generation of partitions

1) Generate time series data of length $N, x(i)$ for the nominal state.

2) Generate complex analytic function using Hilbert transform $x 0(i)=\operatorname{Hilbert}[x(i)]$

3) Arrange abs $(\boldsymbol{x} \mathbf{0})$ and angle $(\boldsymbol{x} \mathbf{0})$ in ascending orders and store the sorted series as $x 1(i)$ and $x 2(i)$.

4) Select number of states $N_{11}$ and $N_{12}$ for $x 1$ and $x 2$, respectively, and assign one symbol to each partition (alphabet).

5) Assign $N_{21}=\left\lfloor\frac{N}{N_{1}}\right\rfloor$ and $N_{22}=\left\lfloor\frac{N}{N_{2}}\right\rfloor$ where \lfloor\rfloor denotes the nearest lower integer.

6) Assign partition1(1) $=x 1(1)$ and partition $1(i+1)=x 1\left(i * N_{2}\right)$ for $1 \leq i \leq\left(N_{11}-1\right)$ and partition $\left(N_{11}+1\right)=x 1(N)$.

7) Assign partition $2(1)=x 2(1)$ and partition $2(i+1)=x 2\left(i * N_{2}\right)$ for $1 \leq i \leq\left(N_{12}-1\right)$ and partition $\left(N_{12}+1\right)=x 2(N)$.

Construction of state probability vector for nominal state

1) Initialize state probability vector $p(i)=0$ for $1 \leq i \leq N_{11} * N_{12}$

2) for $j=1: N$

for $i 1=1: N_{11}-1$

for $i 2=1: N_{12}-1$

if $x 1(j) \geq \operatorname{partition} 1(i 1)$ and $x 1(j)<\operatorname{partition} 1(i 1+1)$ and $x 2(j) \geq \operatorname{partition} 2(i 2)$ and $x 2(j)<\operatorname{partition} 2(i 2+1)$

$p\left[(i 1-1) *\left(N_{12}-1\right)+i 2\right]=p\left[(i 1-1) *\left(N_{12}-1\right)+i 2\right]+1$ end 
Table (Continued.)

\begin{tabular}{l} 
end \\
end \\
3) $p 0(i)=p(i) / N$ for $1 \leq i \leq N_{11} * N_{12}$ \\
\hline \hline Construction of state probability vector for other states \\
\hline \hline 1) Generate time series data of length $N^{\prime}, x(i)$ for the non-nominal state. \\
2) Generate complex analytic function using Hilbert transform \\
$x 0(i)=$ Hilbert $[x(i)]$. \\
3) Arrange abs $(x \mathbf{0})$ and angle $(x \mathbf{0})$ in ascending orders and store the sorted \\
series as $x 1(i)$ and $x 2(i)$. \\
4) Initialize state probability vector $p(i)=0$ for $1 \leq i \leq N_{11} * N_{12}$ \\
5) for $j=1: N^{\prime}$ \\
for $i 1=1: N_{11}-1$ \\
$\quad$ for $i 2=1: N_{12}-1$ \\
$\quad$ if $x 1(j) \geq$ partition $1(i 1)$ and $x 1(j)<$ partition $1(i 1+1)$ and \\
$x 2(j) \geq$ partition $2(i 2)$ and $x 2(j)<$ partition $2(i 2+1)$ \\
$\quad p\left[(i 1-1) *\left(N_{12}-1\right)+i 2\right]=p\left[(i 1-1) *\left(N_{12}-1\right)+i 2\right]+1$ \\
$\quad$ end \\
end $\quad$ \\
6) $p 1(i)=p(i) / N^{\prime}$ for $1 \leq i \leq N_{11} * N_{12}$ \\
\hline \hline
\end{tabular}

Use the same partitions for all the states.

Determination of Anomaly Measure

8) Compute anomaly measure $M=\operatorname{Cos}^{-1} \frac{p_{0} \cdot p_{1}}{\left\|p_{0}\right\|\left\|p_{1}\right\|}$

\section{Acknowledgments}

This work was partially sponsored by Defence Research and Development Organisation, Government of India. R. Chaudhari acknowledges the financial support from Ministry of Human Resource Development, Government of India through Quality Improvement Programme.

\section{References}

[1] De Zilwa, S. R. N., Uhm, J. H., and Whitelaw, J. H., "Combustion Oscillations Close to the Lean Flammability Limit," Combustion Science and Technology, Vol. 160, No. 1, 2000, pp. 231-258. doi:10.1080/00102200008935804

[2] Chaudhuri, S., and Cetegen, B. M., "Blowoff Characteristics of Bluff Body Stabilized Conical Premixed Flames Under Upstream Mixture Gradients and Velocity Oscillations," Combustion and Flame, Vol. 153, No. 4, 2008, pp. 616-633.

doi:10.1016/j.combustflame.2009.10.020

[3] Chaudhuri, S., and Cetegen, B. M., "Blowoff Characteristics of Bluff Body Stabilized Conical Premixed Flames in a Duct with Upstream Mixture Gradients and Velocity Oscillations," Combustion Science and Technology, Vol. 181, No. 4, 2009, pp. 555-569. doi:10.1080/00102200802631278

[4] Chaudhuri, S., Kostska, S., Renfro, M. W., and Cetegen, B. M., "Blowoff Dynamics of Bluff Body Stabilized Turbulent Premixed Flames," Combustion and Flame, Vol. 157, No. 4, 2010, pp. 790-802. doi:10.1016/j.combustflame.2009.10.020

[5] Stöhr, M., Boxx, I., Carter, C., and Meier, W., "Dynamics of Lean Blowout of a Swirl-Stabilized Flame in a Gas Turbine Model Combustor," Proceedings of the Combustion Institute, Vol. 33, No. 2, 2011, pp. 2953-2960. doi:10.1016/j.proci.2010.06.103

[6] Nair, S., Muruganandam, T. M., Olsen, R., Meyers, A., Seitzman, J., Zinn, B. T., and Lieuwen, T., "Lean Blowout Detection in a Single Nozzle Swirl Cup Combustor," 42nd AIAA Aerospace Sciences Meeting, Reno, NV, AIAA Paper 2004-0138, 2004.

[7] Muruganandam, T. M., Nair, S., Scarborough, D., Neumeier, Y., Jagoda, J., Lieuwen, T., Seitzman, J., and Zinn, B. T., "Active Control of Lean Blowout for Turbine Engine Combustors," Journal of Propulsion and Power, Vol. 21, No. 5, 2005, pp. 807-815. doi: $10.2514 / 1.7254$

[8] Nair, S., and Lieuwen, T., "Acoustic Detection of Blowout in Premixed Flames," Journal of Propulsion and Power, Vol. 21, No. 1, 2005, pp. 32-39. doi: $10.2514 / 1.5658$

[9] Prakash, S., Nair, S., Muruganandam, T. M., Neumeier, Y., Lieuwen, T., Seitzman, J. M., and Zinn, B. T., "Acoustic Based Rapid Blowout
Mitigation in a Swirl Stabilized Combustor," ASME Turbo Expo, Reno, NV, ASME Paper GT2005-68589, 2005.

[10] Yi, T., and Gutmark, E. J., "Real-Time Prediction of Incipient Lean Blowout in Gas Turbine Combustors," AIAA Journal, Vol. 45, No. 7, 2007, pp. 1734-1739. doi:10.2514/1.25847

[11] Ray, A., "Symbolic Dynamic Analysis of Complex Systems for Anomaly Detection," Signal Processing, Vol. 84, No. 7, 2004, pp. $1115-1130$. doi:10.1016/j.sigpro.2004.03.011

[12] Tang, T. Z., Tracy, E. R., Boozer, A. D., de Brauw, A., and Brown, R., "Symbol Sequence Statistics in Noisy Chaotic Signal Reconstruction," Physical Review E, Vol. 51, No. 5, 1995, pp. 3871-3889. doi:10.1103/PhysRevE.51.3871

[13] Daw, C. S., Finney, C. E. A., and Tracy, E. R., "A Review of Symbolic Analysis of Experimental Data," Review of Scientific Instruments, Vol. 74, No. 2, 2003, pp. 915-930. doi:10.1063/1.1531823

[14] Rajagopalan, V., and Ray, A., "Symbolic Time Series Analysis via Wavelet-Based Partitioning," Signal Processing, Vol. 86, No. 11, 2006 , pp. 3309-3320. doi:10.1016/j.sigpro.2006.01.014

[15] Subbu, A., and Ray, A., "Space Partitioning via Hilbert Transform for Symbolic Time Series Analysis," Applied Physics Letters, Vol. 92, No. 8,2008 , p. 084107 doi:10.1063/1.2883958

[16] Sarkar, S., Mukherjee, K., and Ray, A., "Generalization of Hilbert Transform for Symbolic Analysis of Noisy Signals," Signal Processing, Vol. 89, No. 6, 2009, pp. 1245-1251. doi:10.1016/j.sigpro.2008.12.009

[17] Jin, X., Gupta, S., Mukherjee, K., and Ray, A., "Wavelet-Based Feature Extraction Using Probabilistic Finite State Automata for Pattern Classification," Pattern Recognition, Vol. 44, No. 7, 2011, pp. 1343 1356. doi:10.1016/j.patcog.2010.12.003

[18] Gupta, S., Ray, A., and Keller, E., "Symbolic Time Series Analysis of Ultrasonic Data for Early Detection of Fatigue Damage," Mechanical Systems and Signal Processing, Vol. 21, No. 2, 2007, pp. 866-884. doi:10.1016/j.ymssp.2005.08.022

[19] Chakraborty, S., Keller, E., Talley, J., Srivastav, A., Ray, A., and Kim, S. "Void Fraction Measurement in Two-Phase Processes via Symbolic Dynamic Filtering of Ultrasonic Signals," Measurement Science and Technology, Vol. 20, No. 2, 2009, p. 023001. doi:10.1088/0957-0233/20/2/023001

[20] Bhambare, K. S., Gupta, S., Mench, M. M., and Ray, A., "A Carbon Monoxide Sensor in Polymer Electrolyte Fuel Cells Based on Symbolic Dynamic Filtering," Sensors and Actuators B, Vol. 134, No. 2, 2008, pp. 803-815. doi:10.1016/j.snb.2008.06.057

[21] Chakraborty, S., Ray, A., Subbu, A., and Keller, E., "Analytic Signal Space Partitioning and Symbolic Dynamic Filtering for Degradation Monitoring of Electric Motors," Signal, Image and Video Processing, Vol. 4, No. 4, 2010, pp. 399-403. doi:10.1007/s11760-009-0133-4

[22] Gupta, S., Ray, A., and Mukhopadhyay, A., "Anomaly Detection in Thermal Pulse Combustors Using Symbolic Time Series Analysis," Journal of Systems and Control Engineering, Vol. 220, No. 5, 2006 pp. 339-351. doi:10.1243/09596518JSCE256

[23] Datta, S., Mukhopadhyay, A., and Sanyal, D., "Use of Temporal Irreversibility of Symbolic Time Series for Early Detection of Extinction in Thermal Pulse Combustors," Proceedings of IMECE2006 2006 ASME International Mechanical Engineering Congress and Exposition, ASME Paper IMECE-2006-16249, Chicago, Nov. 2006.

[24] Chakraborty, S., Gupta, S., Ray, A., and Mukhopadhyay, A., "Dynamic Data-Driven Detection and Estimation of Faults in Thermal Pulse Combustors," Journal of Aerospace Engineering, Vol. 222, No. 8, 2008, pp. 1097-1108. doi:10.1243/09544100JAERO432

[25] Daw, C. S., Kennel, M. B., Finney, C. E. A., and Connolly, F. T., "Observing and Modeling Nonlinear Dynamics in an Internal Combustion Engine," Physical Review E, Vol. 57, No. 3, 1998, pp. 2811-2819. doi:10.1103/PhysRevE.57.2811

[26] Green, J. B., Jr., Daw, C. S., Armfield, J. S., Finney, C. E. A., and Durbetaki, P., "Time Irreversibility of Cycle-by-Cycle Engine Combustion Variations," Proceedings of the 1998 Spring Technical Meeting of the Central States Sections of the Combustion Institute, Lexington, KY, 1998. 
[27] Flynn, T., Bailey, R., Fuller, T., Daw, S., Finney, C., and Stallings, J., "Flame Monitoring Enhances Burner Management," Power Engineering, Vol. 107, No. 2, 2003, pp. 50-54.

[28] Williams, T. C., Schefer, R. W., Oefelein, J. C., and Shaddix, C. R., "Idealized Gas Turbine Combustor for Performance Research and Validation of Large Eddy Simulations," Review of Scientific Instruments, Vol. 78, No. 3, 2007, pp. 035114. doi:10.1063/1.2712936

[29] Meier, W., Weigand, P., Duan, X. R., and Giezendanner-Thobben, R., "Detailed Characterization of the Dynamics of Thermoacoustic Pulsations in a Lean Premixed Swirl Flame," Combustion and Flame, Vol. 150, Nos. 1-2, 2007, pp. 2-26. doi:10.1016/j.combustflame.2007.04.002

[30] Chaudhari, R. R., "Investigation of Thermoacoustic Instabilities and Lean Blowout in a Model Gas Turbine Combustor," Ph.D. Thesis, Jadavpur Univ., Kolkata, India, 2011.
[31] Turns, S. R., An Introduction to Combustion, 2nd ed., McGraw-Hill, New York, 2000, pp. 153-158.

[32] Adenis, P., Mukherjee, K., and Ray, A., "State Splitting and State Merging in Probabilistic Finite State Automata," Proceedings of the American Control Conference, San Francisco, CA, IEEEExplore, 2011, pp. 5145-5150.

[33] Rao, C., Ray, A., Sarkar, S., and Yasar, M., "Review and Comparative Evaluation of Symbolic Dynamic Filtering for Detection of Anomaly Patterns," Signal, Image and Video Processing, Vol. 3, No. 2, May 2009, pp. 101-114.

doi:10.1007/s11760-008-0061-8

J. Seitzman

Associate Editor 
This article has been cited by:

1. Vineeth Nair, Gireehkumaran Thampi, Sulochana Karuppusamy, Saravanan Gopalan, R. I. Sujith. 2013. Loss of chaos in combustion noise as a precursor of impending combustion instability. International Journal of Spray and Combustion Dynamics 5:4, 273-290. [CrossRef] 